\title{
Refractory necrotizing scleritis successfully treated with adalimumab
}

\author{
Lola E. Lawuyi ${ }^{{ }^{*}}$ and Avinash Gurbaxani ${ }^{2}$
}

\begin{abstract}
Necrotizing scleritis is the most severe and destructive form of scleritis with vision-threatening sequelae. It is divided into with inflammation and without inflammation (scleromalacia perforans). Adalimumab is a tumour necrosis factor (TNF)-inhibiting anti-inflammatory medication licensed for the treatment of rheumatoid and psoriatic arthritis, ankylosing spondylitis and inflammatory bowel disease (in the USA). We report two cases of necrotizing scleritis successfully treated with adalimumab.
\end{abstract}

Keywords: Necrotizing scleritis, TNF inhibitors, Adalimumab

\section{Case report}

\section{Case 1}

A 40-year-old lady presented with a history of scleritis for which she was on oral prednisolone for over 1 year with doses ranging from 10 to $80 \mathrm{mg}$ per day. She had pain and discomfort. Her visual acuity was $6 / 6$ and posterior segment was normal. Examination revealed an area of scleral necrosis.

As her scleritis was active, she was commenced on prednisolone $80 \mathrm{mg}$ and mycophenolate mofetil (MMF) (Cellcept) $500 \mathrm{mg} \mathrm{BD}$, increasing to $750 \mathrm{mg}$ BD. However, she had severe gastric discomfort and nausea and was unable to continue with MMF. She was subsequently put on tacrolimus (Prograf) $7.5 \mathrm{mg}$ but developed nausea and lethargy on this. She was unable to reduce oral steroids whilst oral immunosuppression was tried. She was then started on adalimumab (Humira) subcutaneous $40 \mathrm{mg}$ fortnightly and methotrexate $12.5 \mathrm{mg} \mathrm{OD}$ and achieved resolution and control of scleral necrosis after 4 weeks. During the course of treatment, she developed one episode of gastroenteritis during which she missed her Humira. At 9-month follow-up, the disease has remained quiet on two weekly subcutaneous adalimumab treatments.

* Correspondence: Lola27law@yahoo.co.uk

${ }^{1}$ Gate 17, Villa 970 Al Raha Gardens, Abu Dhabi, United Arab Emirates Full list of author information is available at the end of the article

\section{Case 2}

A 52-year-old lady with known rheumatoid arthritis and refractory necrotizing scleritis poorly controlled with oral prednisolone $10-80 \mathrm{mg}$ and oral methotrexate $15 \mathrm{mg}$ once a week was referred for treatment. Her visual acuity was $6 / 9$ in both eyes. Slit lamp examination revealed acute scleral necrosis and impending perforation as shown in Figs. 1 and 2 .

Her past medical history included one episode of scleral perforation to the left eye treated with amniotic patch graft. The patient was started on two weekly subcutaneous adalimumab $40 \mathrm{mg}$ and achieved resolution and control of scleral necrosis after 8 weeks. At 6-month follow-up, the disease has remained quiet on two weekly subcutaneous adalimumab treatments (Fig. 3).

Side effects during treatment included one episode of shingles.

\section{Discussion}

Necrotizing scleritis represents $10-15 \%$ of cases of anterior scleritis and is the most severe [1]. Left untreated, necrotizing scleritis has a rapid and destructive course that can be sight threatening.

Conventional treatment for recurrent or severe cases of necrotizing scleritis involves immunosuppression therapy and high-dose oral steroids [2]. Side effects associated with these treatments and a poor response may require patients to terminate treatment (as did our two patients), making this condition very difficult to manage. 


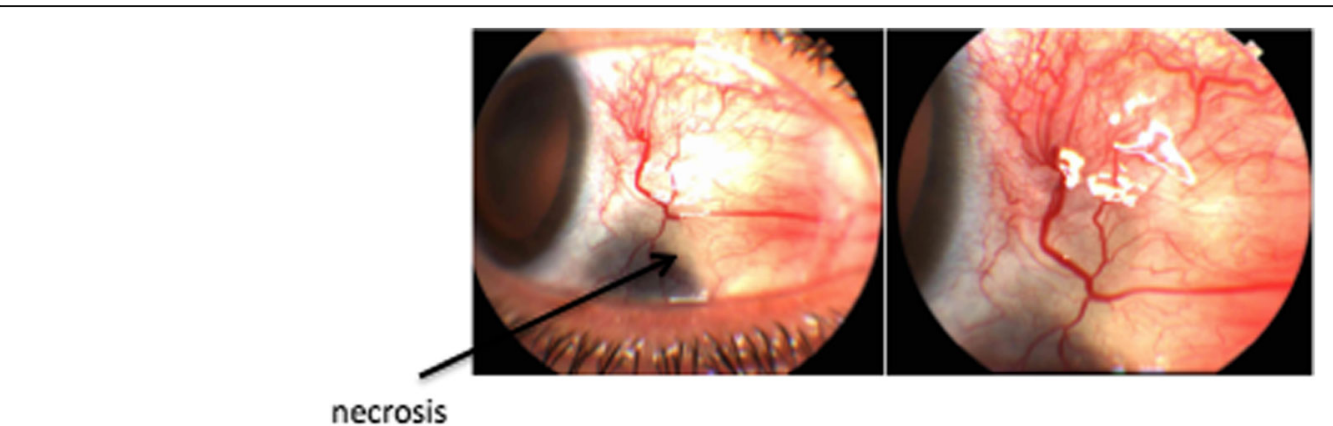

Fig. 1 Scleral necrosis RE

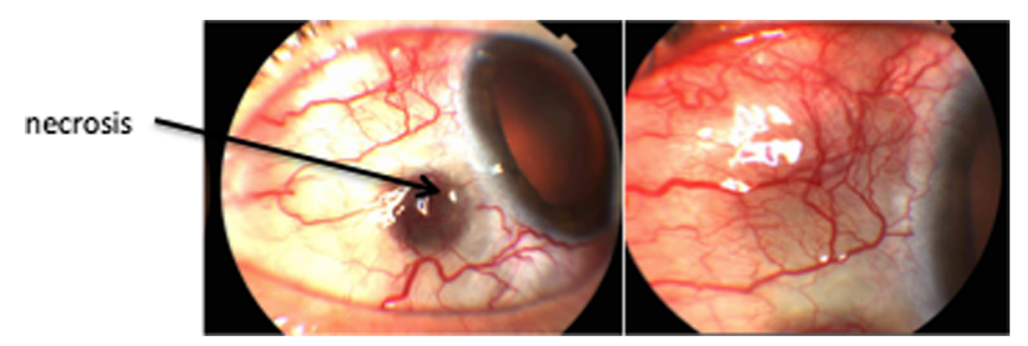

Fig. 2 Scleral necrosis LE
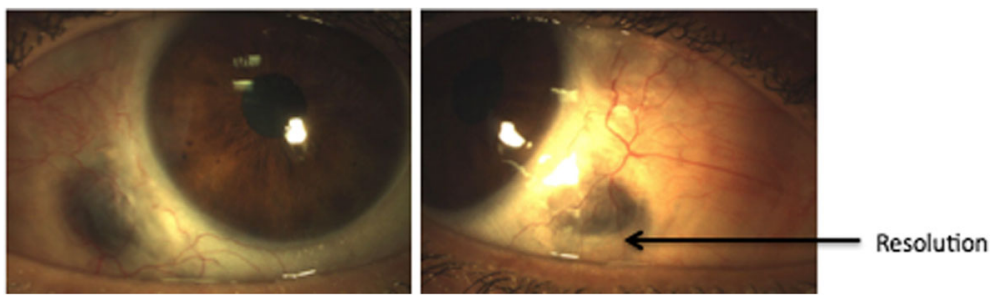

Fig. 3 Resolution/control on adalimumab at 6-month follow-up 
The predominant cytokine TNF-alpha induces the matrix metalloproteinases (MMPs) enzymes, MMP-3 and MMP-9 [3]. These are predominantly responsible for the destruction of the scleral wall and adjacent cornea in severe scleritis, particularly associated with necrosis [4].

Infliximab (Remicade Centocor, Johnson \& Johnson, USA) and adalimumab (Humira, Abbott Laboratories, Chicago, IL) are monoclonal antibodies that recognise, bind to and inhibit TNF, thereby decreasing inflammation.

Infliximab has shown long-term efficacy in the treatment of refractory posterior uveitis [5]. Published literature has reported improvement in patients with either idiopathic scleritis or scleritis associated with systemic disease treated with infliximab [6].

Ragam et al. evaluated the use of TNF inhibitors infliximab and adalimumab in 17 patients with non-infectious and non-necrotizing scleritis and achieved control of active inflammation for at least 2 months in $15(88 \%)$ of 17 patients [7].

Adalimumab has been reported as successful in the treatment of bilateral idiopathic nodular scleritis (with no side effects reported) [8] and nodular scleritis associated with RA [9].

Morarji et al. reported the successful treatment of necrotizing scleritis (secondary to granulomatosis with polyangiitis) with infliximab whilst waiting for rituximab to induce disease remission [10]. Two intravenous 1-g doses of rituximab (MabThera, Hoffmann-La Roche, Ltd.) (a chimeric murine and human monoclonal antibody directed against the $\mathrm{CD} 20$ antigen [11]) were used, given 2 weeks apart. However after 3 weeks, the necrotizing scleritis was still active. Therefore, infusions of infliximab (Remicade, Merck \& Co.) $5 \mathrm{mg} / \mathrm{kg}$ doses at baseline, 2, 6 and 12 weeks were given, controlling the necrotizing scleritis by week 6 . This enabled cyclophosphamide to be avoided in the acute phase (as rituximab can take up to 6 months to work).

In this case series, we report two cases of refractory necrotizing scleritis that were successfully treated with adalimumab. During treatment, one patient developed gastroenteritis and the other developed shingles.

Known potential side effects have been documented in clinical trials and postmarket surveillance with TNF inhibitor use. The development of injection site reactions [12], infusion reactions [12], and the reactivation of latent tuberculosis [13] is increased with TNF inhibitor use. Therefore, proper screening of patients with the tuberculin skin test and chest $\mathrm{x}$-ray and/or interferon gamma assay should be performed prior to initiating these therapies. Other side effects include the development of demyelinating disease [14] and heart failure [15].

In conclusion, despite the risks involved with TNF inhibitor use, with adequate screening of patients, the present case series highlights that adalimumab offers a new tool for the treatment of refractory necrotizing scleritis when conventional treatments are contraindicated or have failed.

\section{Acknowledgements}

We thank the photography department at Moorfields Eye Hospital Dubai for help in preparation of the images. All persons who have made substantial contributions to the work reported in the manuscript (e.g., technical help, writing and editing assistance, general support), but who do not meet the criteria for authorship, are named in the Acknowledgements and have given us their written permission to be named.

\section{Authors' contributions}

All persons who meet authorship criteria are listed as authors, and all authors certify that they have participated sufficiently in the work to take public responsibility of this manuscript. Furthermore, each author certifies that this material or similar material has not been and will not be submitted to or published in any other publication before its appearance in the Journal of Ophthalmic Inflammation and Infection. All authors read and approved the final manuscript.

\section{Competing interests}

The authors declare that they have no competing interests.

\section{Author details}

${ }^{1}$ Gate 17, Villa 970 Al Raha Gardens, Abu Dhabi, United Arab Emirates. ${ }^{2}$ Moorfields Eye Hospital Dubai, Medical Retinal and Cataract, Dubai, United Arab Emirates.

Received: 12 April 2016 Accepted: 29 September 2016

Published online: 12 October 2016

References

1. Nussenbalt RB, Whitcup SM (2010) Uveitis: fundamentals and clinical practice, 4th edn. Elsevier, Philadelphia

2. Garg SJ. Wills Eye Institute- Uveitis (Colour Atlas and Synopsis of Clinical Ophthalmology). Lippincott Williams \& Wilkins; 2012

3. Di Girolamo N, Visvanathan K, Lloyd A, Wakefield D (1997) Expression of TNF-alpha by human plasma cells in chronic inflammation. J Leukoc Biol 61(6):667-678

4. Di Girolamo N, Lloyd A, McCluskey P, Filipic M, Wakefield D (1997) Increased expression of matrix metalloproteinases in vivo in scleritis tissue and in vitro in cultured human scleral fibroblasts. Am J Pathol 150(2):653-666

5. Lopez Gonzalez R, Loza E, Jover JA et al (2009) Treatment for refractory posterior uveitis with infliximab: a 7-year follow-up study. Scand J Rheumatol 38(1):58-62

6. Sen HN, Sangave A, Hammel K et al (2009) Infliximab for the treatment of active scleritis. Can J Ophthalmol 44(3):e9-e12

7. Ragum A, Kolomeyer AM, Fang C, Xu Y, Chu DS (2014) Treatment of chronic, noninfectious, nonnecrotizing scleritis with tumor necrosis factor alpha inhibitors. Ocul Immunol Inflamm 22(6):469-477

8. Bawazeer AM, Raffa LH (2011) Adalimumab in the treatment of recurrent idiopathic bilateral nodular scleritis. Oman J Ophthalmol 4(3):139-141

9. Restrepo JP, Molina MP (2010) Successful treatment of severe nodular scleritis with adalimumab. Clin Rheumatol 29(5):559-561

10. Morarji J, Joshi L, Tomkins-Netzer O, Lightman S, Taylor SRJ (2012) Combined infliximab and rituximab in necrotising scleritis. Case Rep Ophthalmol 3:286-290

11. Blank M, Shoenfeld $Y$ (2007) B cell targeted therapy in autoimmunity. J Autoimmun 28:62-68

12. Ruderman EM (2012) Overview of safety of non-biologic and biologic DMARDs. Rheumatology 51(Suppl 6):vi37-vi43

13. Keane J, Gershon S, Wise RP et al (2001) Tuberculosis associated with infliximab, a tumor necrosis factor alpha-neutralizing agent. N Engl J Med 345(15):1098-1104

14. Solomon AJ, Spain RI, Kruer MC et al (2011) Inflammatory neurological disease in patients treated with tumor necrosis factor alpha inhibitors. Mult Scler 17(12):1472-1487

15. British Society for Rheumatology (2005) Update on the British Society for Rheumatology. Rheumatology guidelines for prescribing TNFa blockers in adults with rheumatoid arthritis (update of previous guidelines of April 2001). Rheumatology 44(2):157-163, [Online] [Access 2015 November]. Available from URL http://rheumatology.oxfordjournals.org 